The normalized expression levels are combined to one numeric value which determines the drug susceptibility of the investigated strain

We successfully developed 8 primary sets of RNA biomarkers for ten $1^{\text {st }}$-line, $2^{\text {nd }}$-line and new drugs. Taking isoniazid as proof of principle, we present a biomarker set of 5 responsive genes and 3 normalizing genes, which enables to distinguish susceptible, low- and high resistant TB strains after 6 hours incubation. Next, preliminary results demonstrate that the biomarker sets can successfully discriminate between susceptible and resistance strains for the selected drugs.

We present a robust, RNA-based DST without the need for RNA extraction. The assay was proven to be efficient for isoniazid. With a total of 8 biomarker sets under optimization, the drug resistance profile of up to 14 drugs can be determined.

\subsection{9} doi: 10.15789/2220-7619-2018-4-6.59

\section{POPULATION STRUCTURE OF MYCOBACTERIUM TUBERCULOSIS IN RUSSIAN REGIONS BORDERING EU COUNTRIES}

A. Vyazovaya ${ }^{1}$, N. Solovieva ${ }^{2}$, A. Gerasimova ${ }^{1}$

G. Akhmedova ${ }^{3}$, E. Turkin ${ }^{3}$, T. Sunchalina ${ }^{4}$, R. Tarashkevich ${ }^{5}$, S. Bogatin ${ }^{5}$, N. Gavrilova ${ }^{6}$, A. Bychkova ${ }^{6}$, E. Anikieva ${ }^{6}$, V. Zhuravlev ${ }^{2}$, O. Narvskaya ${ }^{1,2}$, I. Mokrousov ${ }^{1}$

${ }^{1}$ St. Petersburg Pasteur Institute, St. Petersburg, Russia; ${ }^{2}$ Research Institute of Phthisiopulmonology, St. Petersburg, Russia; ${ }^{3}$ Regional Tuberculosis Dispensary, Kaliningrad, Russia $:{ }^{4}$ Republican

Tuberculosis Dispensary, Petrozavodsk, Karelia, Russia; ${ }^{5}$ Regional Tuberculosis Dispensary, Pskov, Russia; ${ }^{6}$ Regional Tuberculosis

Dispensary, Murmansk, Russia

Population structure of Mycobacterium tuberculosis in Russia is characterized by predominance of the Beijing genotype strains. A high burden of multidrug-resistant (MDR) tuberculosis in the northwestern Russia may have a negative impact on TB control programs in the neighboring countries of the European Union.

We aimed to assess the population structure of M. tuberculosis in northwestern Russian regions (Karelia, Kaliningrad, Murmansk, Pskov) bordering countries of the European Union (Finland, Norway, Estonia, Latvia, Lithuania and Poland).

A total of $304 M$. tuberculosis isolates (2013-2017) from newly diagnosed TB patients in geographically distant regions of northwestern Russia were studied: Republic of Karelia $(n=78)$, Pskov $(n=89)$, Kaliningrad $(n=73)$ and Murmansk provinces $(n=66)$. The Beijing genotype family and its particular clusters B0/W148 and 94-32 were detected by analysis of specific markers in $\mathrm{dnaA}$ dnaN::IS6110, Rv2664-Rv2665::IS6110 and sigE98, respectively. Non-Beijing isolates were subjected to spoligotyping followed by comparison to the SITVIT_WEB.

These 4 Russian regions differed to some extent in the prevalence of the Beijing genotype: from to $55.1 \%$ in Karelia, $44.9 \%$ in Pskov, $63.0 \%$ in Kaliningard and $51.5 \%$ in Murmansk. Beijing B0/W148-cluster was identified in $17.9 ; 6.7 ; 19.2$ and $10.6 \%$ isolates, respectively. The prevalence of the Beijing 94-32-cluster did not differ: $28.2 ; 29.2 ; 28.8$ and $30.3 \%$, respectively. In Pskov and Murmansk, most strains were drug susceptible (62.9 and $62.1 \%$ ), while $17.9 \%$ and $25.8 \%$ were MDR, respectively. In contrast, MDR strains prevailed in Karelia and Kaliningard - 51.3 and $43.8 \%$, while 41.0 and $36.9 \%$ strains were susceptible. In total, for four regions together, 90.2\% (37/41) strains of the Beijing B0/W148-cluster were MDR. Beijing 94-32-cluster strains showed much lower rate of MDR - 34.8\%, (31/89) ( $\mathrm{P}<0.0001)$, while $41.6 \%$ (37/89) were susceptible. Among non-Beijing M. tuberculosis the largest families were T (14.1 and $15.1 \%)$ in Karelia and Kaliningard, LAM (23.6\%) in Pskov, and Ural (19.7\%) in Murmansk.

M. tuberculosis population in the northwestern Russian-EU border provinces is marked by predominance of the major clonal complexes of the Beijing genotype (B0/W148 и 94-32). However, these clones significantly differed in the proportion of MDR. A circulation of the MDR-associated isolates of the Beijing B0/W148-cluster presents the major concern for local and national TB control programs in the Russian regions and likely adverse impact on the neighboring EU countries.

Funding: Russian Science Foundation grant 14-14-00292.

6.60 doi: 10.15789/2220-7619-2018-4-6.60

\section{DRUG RESISTANCE IN MYCOBACTERIUM} TUBERCULOSIS: FROM PHENOTYPIC MIC-ANALYSIS TO WGS FOR ROUTINE DRUG SUSCEPTIBILITY TESTING

\section{J. Werngren}

Supranational Reference Laboratory for Tuberculosis and Public Health Agency of Sweden, Department of Microbiology, Unit of Laboratory Surveillance of Bacterial Pathogens, Solna, Sweden

The phenotypic methods used for drug susceptibility testing (DST) of Mycobacterium tuberculosis generally take weeks or months and require culture and manipulation of large numbers of highly infectious bacilli. Drug resistance in $M$. tuberculosis is almost exclusively a consequence of genomic mutations and so molecular determination of resistance offers a rapid, potentially cost effective, and safer alternative.

In 2016, the SRL in Stockholm introduced whole genome sequencing (WGS) for the molecular typing of clinical isolates of M. tuberculosis. At the same time, the WGS analysis made information of various putative drug resistance-related mutations available, which was utilized to complement the routine phenotypic DST. Since 2016, the WGS information on resistance to all clinically relevant drugs is analysed and routinely reported together with the extended phenotypic DST results for all the Swedish multidrug resistant (MDR) isolates.

Retrospectively, we have performed an observational study to compare the WGS data to the phenotypic DST to predict the resistance phenotype from the genetic sequence. The study included 877 clinical TB isolates (only one sample per patient was included) received by the Public Health Agency of Sweden between 01.01.2016 and 31.12.2017. The analysis of the isolates' resistance profiles for the first and second line drugs, obtained from the WGS and Bactec MGIT 960, is ongoing.

Simultaneously, we are evaluating the microbroth dilution technique used for the MIC determination of 150 consecutive (MDR) M. tuberculosis together with the corresponding WGS analysis as a part of a collaboration with the CRyPTIC (Comprehensive Resistance Prediction for Tuberculosis: an International Consortium) network. The MIC determinations of drug susceptible and resistant $M$. tuberculosis isolates are necessary in the work to establish correct critical test concentrations for phenotypic DST as well as to identify the minimal, moderate and high confidence mutations. Such coupled analysis will more accurately define clinical drug resistance and may possibly constitute the DST algorithm of choice in an increasing number of laboratories. 